\title{
MODELLING SOLVENT-MEDIATED POLYMORPHIC TRANSITIONS DURING COOLING SOLUTION CRYSTALLIZATION
}

\author{
G. Févotte ${ }^{(*)} \&$ N. Sheibat-Othman
}

\author{
LAGEP UMR CNRS 5007, Université Lyon 1, ESCPE. Bât. 308G, 43 bld. du 11 novembre 1918. 69622 \\ Villeurbanne Cedex. France (email fevotte@lagep.univ-lyon1.fr)
}

\begin{abstract}
Polymorphism is one of the most important issues raised by the industrial crystallization of Active Pharmaceutical Ingredients (API). The dynamic modelling of polymorphic transformations is developed using $\mathrm{Femlab}^{\circledR}$, a multiphysics simulation software, which is applied to the resolution of the Populations Balance Equations (PBE) involved. The stable and metastable distinct populations of crystals are represented in a mono-dimensional framework (i.e. one particle size as internal variable.) The simulation reproduces experimental features associated with Ostwald's Rule of Stages, it allows explaining and analysing the conditions of occurrence of both stable and metastable forms of the solid product. Copyright (C) 2005 IFAC
\end{abstract}

Keywords: Partial differential equations, Dynamic modelling, Particulate processing, Product quality, Chemical industry, Crystallization, Polymorphism, Femlab.

\section{INTRODUCTION}

Many solid substances can appear in two or more solid phases according to the crystallization medium and to the experimental conditions. For a given molecule, the various phases are characterized by different crystal structures and the corresponding solids are referred to as polymorphs. In 2002, about $5 \%$ of all organic molecules referenced in the crystallographic database of Cambridge (i.e. 250000 crystalline structures) were known to exhibit polymorphism (Cambridge, 2002). This number increases rapidly which confirms the increasing theoretical and industrial future issues of polymorphism. Polymorphism influences the properties and applications of the solid particles, especially in the pharmaceutical industry. As far as pharmaceutical products are concerned, bioavailability obviously depends on solubility which is connected to several crystal parameters. A significant lack of efficiency or the occurrence of undesirable secondary effects may also occur as a result of the solid phase transformation of APIs Active Pharmaceutical Ingredients).
In such a context, most difficulties related to the production of the appropriate forms of an API arise from the so-called Ostwald's Rule of Stages (ORS) which was never demonstrated: "In the case of a product capable of crystallizing in several forms, it will be the least stable form which is first produced by spontaneous nucleation, followed successively by forms of increasing stability.” A major practical consequence of the rule, arises from the difficulty in ensuring that the actual form of a given API, obtained at a given time, will not further crystallize in a more stable unknown form. Such uncertainty clearly jeopardizes the production of APIs as the regulatory agencies -such as the FDA- only authorize a specific form of the API to be delivered on the market.

Polymorphism can take place in different ways during a solution crystallization process. After primary nucleation (i.e. the birth of crystal germs) of the metastable solid form, the same form can be obtained at the end of the batch process, which means that no significant nucleation of the stable 
form occurs. For other processes, the more stable known form is obtained in the final suspension: this implies that after its nucleation and growth, the metastable form disappears in favour of the stable one. The transition between the two solid forms is made easier by the mediation of the solvent: during the transition, the metastable crystals dissolve so that the solute concentration can feed the growth of the stable particles.

It is an interesting issue to reproduce, through numerical simulation, the possible different behaviours of such polymorphic systems. Indeed, while the thermodynamic bases of polymorphism are now clearly established (Brittain, 1999), the fundamental dynamic processes involved during polymorphic transitions are far from being explained today. In particular, two major problems still remain unsolved:

- Why, according to ORS, do metastable forms appear first during the crystallization process ?

- What are the dynamic mechanisms and what are the kinetic parameters that could allow one to represent the behaviour of polymorphic systems and to understand why either the stable or the metastable form can be obtained at the end of a given batch crystallization process.

The first point is outside the scope of this work. In the following, it is simply assumed that the kinetic nucleation parameters are such that the system reproduces the ORS. The parameters for primary nucleation will therefore be set such that the metastable form nucleates first (Cardew, 1985). As far as the second question is concerned, only few papers deal with dynamic features of solventmediated phase transition phenomena and no numerical simulations have been reported yet. A model is presented below, which requires the resolution of the Population Balance Equations (PBE) describing the generation and growth of both stable and metastable crystals.

The paper is organized as follows, Part 2 presents the main PDE of the model and the related differential equations, Part 3 gives some indications on the use of Femlab to solve these equations. Part 4 describes briefly the simulation conditions and presents some simulation results which are briefly discussed.

\section{PBEs FOR SOLUTION CRYSTALLIZATION AND KINETIC MODELS FOR NUCLEATION AND GROWTH OF CRYSTALS}

\subsection{Population Balance Equations.}

The population of crystals during the batch process is described by the density function $\psi$. For the sake of simplicity, it is assumed that one size dimension $\mathrm{L}$ is sufficient for the characterisation of the crystals sizes and that the Crystal Size Distribution (CSD) does not depend on spatial coordinates in the wellmixed crystallizer.

If $\psi(\mathrm{L}, \mathrm{t})$.dL is the number density function of crystals of size between $\mathrm{L}$ and $\mathrm{L}+\mathrm{dL}$ per unit volume at time $\mathrm{t}$, then during the interval $\mathrm{dt}$, the balance of crystals in the size interval $[\mathrm{L}, \mathrm{L}+\mathrm{dL}$ ] can be written as follows:

$$
\frac{1}{V_{T}(t)} \frac{\partial}{\partial t}\left[\Psi_{i}(L, t) V_{T}(t)\right]+\nabla\left(G(L, t) \Psi_{i}(L, t)\right)=0
$$

index i refers to the form of the solid: $\psi_{1}$ represents the population of stable crystals, $\psi_{2}$ represents metastable solid particles.

A boundary condition describes the generation (i.e. both primary and secondary nucleation) of new particles in the suspension. Such generation can occur when no solid is present in the crystallizer, through primary nucleation, or when many crystals are already present in the crystallization medium: through secondary nucleation. Again, both stable and metastable germs can appear :

$$
\Psi_{\mathrm{i}}(0, \mathrm{t})=\frac{R n 1_{\mathrm{i}}(\mathrm{t})+\mathrm{Rn} 2_{\mathrm{i}}(\mathrm{t})}{\mathrm{G}_{\mathrm{i}}(0, \mathrm{t})}
$$

where Rn1 and Rn2 are the primary and secondary nucleation rates of the 2 forms, $G$ is the growth rate.

\subsection{Nucleation of stable \& metastable forms.}

As mentioned above, the different forms do not present the same solubility. Thermodynamics clearly shows that increasing stability is always associated to decreasing solubility. Moreover, the two solubility curves can cross (the system is referred to as enantiotropic) or not (monotropic system) in the temperature range of the batch crystallization process.

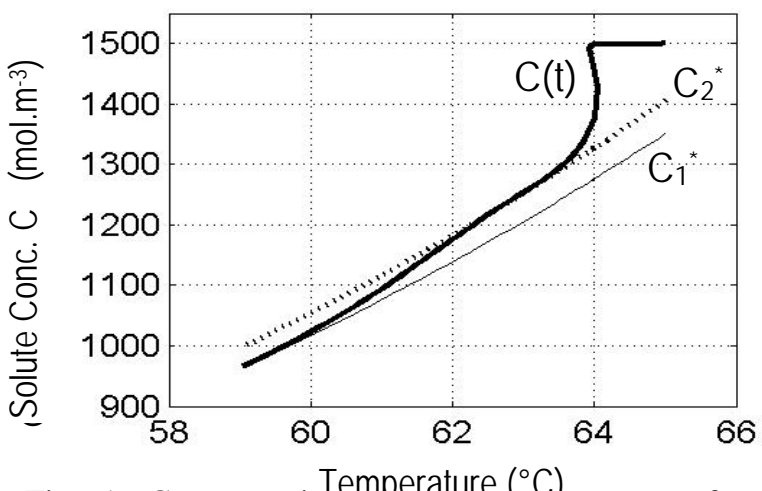

Fig. 1. Concentrati ${ }^{\text {Temperature }}\left({ }^{\circ} \mathrm{C}\right)$ of a monotropic system (i.e. the solubility curves do not cross).

As supersaturation is the driving force of most crystallization phenomena, one has to compute different levels of supersaturation for both forms, with respect to their own solubility. Say :

$$
\sigma_{\mathrm{i}}(\mathrm{t})=\mathrm{C}(\mathrm{t})-\mathrm{C}_{\mathrm{i}}^{*}(\mathrm{t}) \text { and } \beta_{\mathrm{i}}(\mathrm{t})=\mathrm{C}(\mathrm{t}) / \mathrm{C}_{\mathrm{i}}^{*}(\mathrm{t})
$$


where $C_{i}^{*}(t)$ is the solubility of form $i$ at temperature $\mathrm{T}(\mathrm{t}), \mathrm{C}(\mathrm{t})$ is the solute concentration, $\sigma_{\mathrm{i}}$ $\& \beta_{\mathrm{i}}$ are the relative supersaturation and the degree of supersa-turation, respectively. The previous definitions are illustrated by Fig.(1) which represents an monotropic system where form 2 is metastable.

Many kinetic equations are available to represent nucleation phenomena. The complexity of these models obviously depends on the physics which is involved to describe the onset of solid phase (i.e. from purely empirical models to advanced mechanistic models.) The following equations will be used in the sequel:

$$
\begin{aligned}
& \operatorname{Rn}_{\mathrm{i}}(\mathrm{t})=\mathrm{A}_{\mathrm{i}} \exp \left(\frac{-\mathrm{B}_{\mathrm{i}}}{\left(\ln \beta_{\mathrm{i}}(\mathrm{t})\right)^{2}}\right) \\
& \operatorname{Rn}_{\mathrm{i}}(\mathrm{t})=\mathrm{Kn} n_{\mathrm{i}} \cdot \sigma_{\mathrm{i}}(\mathrm{t})^{\mathrm{I}_{\mathrm{i}}} \cdot \mathrm{Cs}_{\mathrm{i}}(\mathrm{t})
\end{aligned}
$$

$A_{i}, B_{i}$ and $K n_{i}$ are kinetic parameters. $C s_{i}$ is the concentration of solid i in suspension. Eq.(5) clearly accounts for the activation of the secondary nucleation of form i by the presence of crystals of the same form.

\subsection{Crystal growth.}

In the mono-dimensional case, the crystal growth rate is simply defined as : $\mathrm{G}_{\mathrm{i}}(\mathrm{t})=\mathrm{dL} / \mathrm{dt}$

where $\mathrm{L}$ is the characteristic size of a given crystal of type i within the size domain. $G$ is the result of a competition between diffusive phenomena and the integration of molecules of solute in the crystal lattice. One therefore has to describe both integration and possible diffusive limitations in the modelling of the growth rate. This can be done through the introduction of an efficiency factor $\eta$ relating the flux of molecules that would be transferred without any diffusive limitation (i.e. the maximal flux), to the real flux. This approach was first proposed by Garside (1971). In the following, it is assumed that the integration mechanism is of

second order: $\quad G_{i}(t)=2 \frac{M}{\rho_{i}} k_{c_{i}} \eta_{i} \sigma_{i}(t)^{2}$

$\mathrm{M}$ is the molecular weight, $\rho_{i}$ is the density of solid $\mathrm{i}$ and $\mathrm{k}_{\mathrm{ci}}$ is a kinetic integration coefficient. $\eta$ is the solution of the following equation:

$$
\left[\left(\mathrm{C}-\mathrm{C}_{\mathrm{i}}^{*}\right)^{\mathrm{n}-1} \mathrm{k}_{\mathrm{C}_{\mathrm{i}}} / \mathrm{k}_{\mathrm{d}}\right] \eta_{\mathrm{i}}+\eta_{\mathrm{i}}^{1 / \mathrm{n}}-1=0
$$

In the sequel, the mass transfer coefficient $k_{d}$ is computed using the correlation of Levins \& Glastonbury (1972) relating the mass transfer coefficient in suspension to the particle size.

\subsection{Energy balance of the crystallizer.}

Cooling a batch industrial crystallizer is generally performed through the use of jacketed reactors. The jacket temperature is denoted by $T_{j}$ below, one assumes the temperature is perfectly controlled (i.e. the setpoint trajectory is ideally tracked).

After primary nucleation, the exothermal effect of crystallization leads to a "bump" in the temperature profile of the slurry. Such thermal effect is continuous, although it is more pronounced after primary nucleation. Actually any slight increase of $\mathrm{T}(\mathrm{t})$ reduces supersaturation and moderates the nucleation kinetics in a highly nonlinear way. So, in addition to the previous equations, the following energy balance was introduced in the model:

$$
\mathrm{mCp} \frac{\mathrm{dT}}{\mathrm{dt}}=\mathrm{HS}\left(\mathrm{T}_{\mathrm{j}}-\mathrm{T}\right)+\mathrm{V}_{\text {susp }} \Delta \mathrm{H}_{\text {crist }} \frac{\mathrm{dC}_{\mathrm{s}}}{\mathrm{dt}}
$$

where $\mathrm{C}_{\mathrm{s}}$ is the total mass of solid (i.e. forms 1 \& $2), H$ is the overall heat transfer coefficient of the jacket and $\mathrm{S}$ is the cooling surface. $\Delta \mathrm{H}_{\text {crist }}$ is the crystallization enthalpy.

\section{SOLVING THE PBEs USING FEMLAB ${ }^{\circledR}$}

\subsection{Brief presentation of Femlab}

In the past 10 years the use of PBEs came out for the modelling of various particulates processes, including crystallization. These equations admit analytical solutions in the simpler cases but generally, the complexity of the crystallisation systems encountered with organic products precludes the existence of analytic solutions and requires the development and adaptation of numerical techniques. From a purely numerical viewpoint, hyperbolic PDEs like Eqs.(1-2) are known to lead to instable solutions which makes them really difficult to solve. Major problems also arise from the 2 different sizes and time steps involved in the modelling (i.e. nucleation phenomena can only be represented using very fine time and scale mappings, while crystal growth requires "normal" integration steps.) Moreover, the PBEs are strongly non linear and their coefficients depend indirectly on density functions, which makes their resolution even more difficult.

FEMLAB is an interactive environment for modelling and simulating scientific and engineering problems based on PDEs. FEMLAB's multiphysics feature allows simultaneous modelling of any combination of phenomena, in particular the PBEs involved during the dynamic modelling of crystallization process. There are two available ways of modelling: ready-to-use applications, which allow to model simply by defining the relevant physical quantities rather than the equations directly and equation-based modelling, which provides the freedom to create specific equations. Both can be combined using multiphysics. 


\subsection{Computation of $C(t)$.}

The solute concentration $\mathrm{C}(\mathrm{t})$ is a key variable in the simulation as it determines the level of supersaturation and, consequently, any nucleation and growth event occurring in the crystallizer. $\mathrm{C}(\mathrm{t})$ is required to solve the PBEs, but also results from the computation: any molecule appearing in the solid phase should be subtracted from the solute.

For both solid phases, the concentration of API can be computed assuming spherical shapes of the particles: $\mathrm{Cs}_{\mathrm{i}}(\mathrm{t})=\frac{\pi \rho_{\mathrm{i}}}{6 \mathrm{M}} \int_{\mathrm{L}_{0}}^{\mathrm{L}_{\mathrm{i} \max }} \Psi_{\mathrm{i}}(\mathrm{L}, \mathrm{t}) \mathrm{L}^{3} \mathrm{dL}$

The mass balance of solute provides the concentration of API remaining at the dissolved state. At any time step, Femlab allows computing and closing the API mass balance through the possible definition of integrated variables such as $\mathrm{C}_{\mathrm{s}}(\mathrm{t})$.

\section{SIMULATION CONDITIONS \& RESULTS}

\subsection{Modelling assumptions.}

- In the following is assumed that the crystallization process obeys Ostwald's Rule of Stages, which means that primary nucleation of form 2 occurs first. One can simulate this feature through the tuning of appropriate kinetic parameters in eq.(4), which is consistent with previous papers (Cardew, 1985)

- As already outlined (see eq.(5)), it is assumed that secondary nucleation of form $i$ is enhanced by contact between the solute and crystals of the same form. This assumption is strongly confirmed by experimental observation (Mersmann, 2002)

- The possibility, for the $\mathrm{C}(\mathrm{t})$ trajectory, of crossing the solubility of form 2 after its primary nucleation, requires that nuclei of form 1 go on growing and generating new nuclei during the growth of form 2 crystals. As soon as the solubility of form 2 has been crossed any nucleation or growth of form 2 becomes impossible and crystals of form 2 can dissolve. Such a solvent-mediated process goes on until the concentration reaches the solubility of form 1. The dissolution of crystals is assumed to follow a first order kinetic law with constant parameters :

$$
\mathrm{D}_{2}(\mathrm{t})=\mathrm{k}_{\text {dissol }} \sigma_{2}(\mathrm{t})
$$

\subsection{Realistic parameter values.}

Data for a realistic simulation of the industrial crystallization of organic molecules were taken from Marchal's Thesis (1989). However, the parameters for primary nucleation were tuned artificially so as to reproduce various situations according to ORS. The solubility curve of the stable form is the same as the solubility of adipic acid. The solubility of the metastable particles is obtained through a slight translation of the first solubility, as one can see in Fig.1. Therefore, a monotropic system is simulated in the sequel. The following parameters were introduced as references:

$\mathrm{A}_{1}=3.10^{13} \mathrm{nb} \cdot \mathrm{m}^{-3} \cdot \mathrm{s}^{-1}, \mathrm{~B}_{1}=0.65 ; \mathrm{A}_{2}=8.10^{14} \mathrm{nb} \cdot \mathrm{m}^{-3} \cdot \mathrm{s}^{-1}$ $\mathrm{B}_{2}=0.36$

A cooling profile for the jacket temperature $T_{j}(t)$ is applied with constant slope $: \mathrm{dT}_{\mathrm{j}} / \mathrm{dt}=-0.006 \mathrm{~K} / \mathrm{s}$, the initial temperature is $65^{\circ} \mathrm{C}$. The initial solute concentration is 1500 mol. $\mathrm{L}^{-1}$.

\subsection{Simulation of batch crystallization with final production of the stable form.}

In order to reproduce such experimental behaviour, the previous reference nucleation data $(A, B)$ are set. Here, the primary nucleation of stable form does not contradict ORS, but the stable nuclei are enough to allow crossing $\mathrm{C}_{2}^{*}(\mathrm{t})$. Fig. 1 displays the concentration profile during batch process. Though few experimental trajectories of this kind were reported in the literature, the results correspond to previously reported experimental data (Fevotte, 2002).

Fig.2 shows the two supersaturation profiles and Fig. 3 presents the time-variations of the ratio between stable and metastable solids. It clearly appears that the metastable form dominates first and that the phase transition is achieved at the end of the batch.

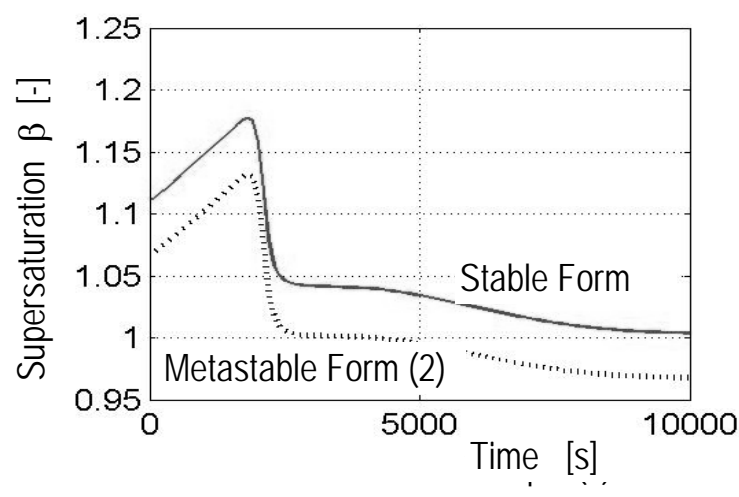

Fig. 2. Simulated supersaturation profiles during batch cooling crystallization with solventmediated polymorphic transition.

Fig.4 shows that the metastable particle number increases dramatically after primary nucleation and reaches a plateau during which the supersaturation level is almost null with respect to form 2. The dissolution of form 2 to the benefit of form 1 then follows, until the end of the batch process.

The evolutions of the Crystals Size Distributions (CSD) of the 2 forms are presented in Fig.5 which reproduces the appearance and growth of metastable crystals followed by their dissolution, 
together with the generation of crystals in the stable form.

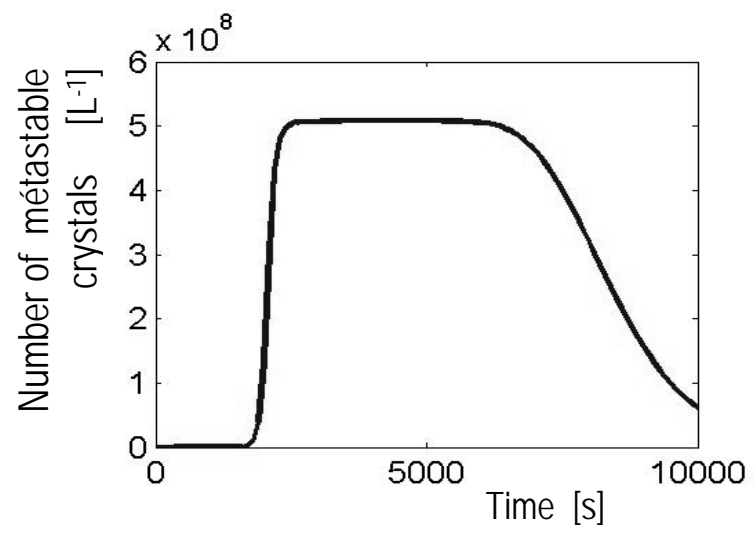

Fig. 3. Time variations of the solid, $\mathrm{t}_{0}$ indicates the onset of primary nucleation.

Fig. 4. Time variations of the total number of metastable particles.

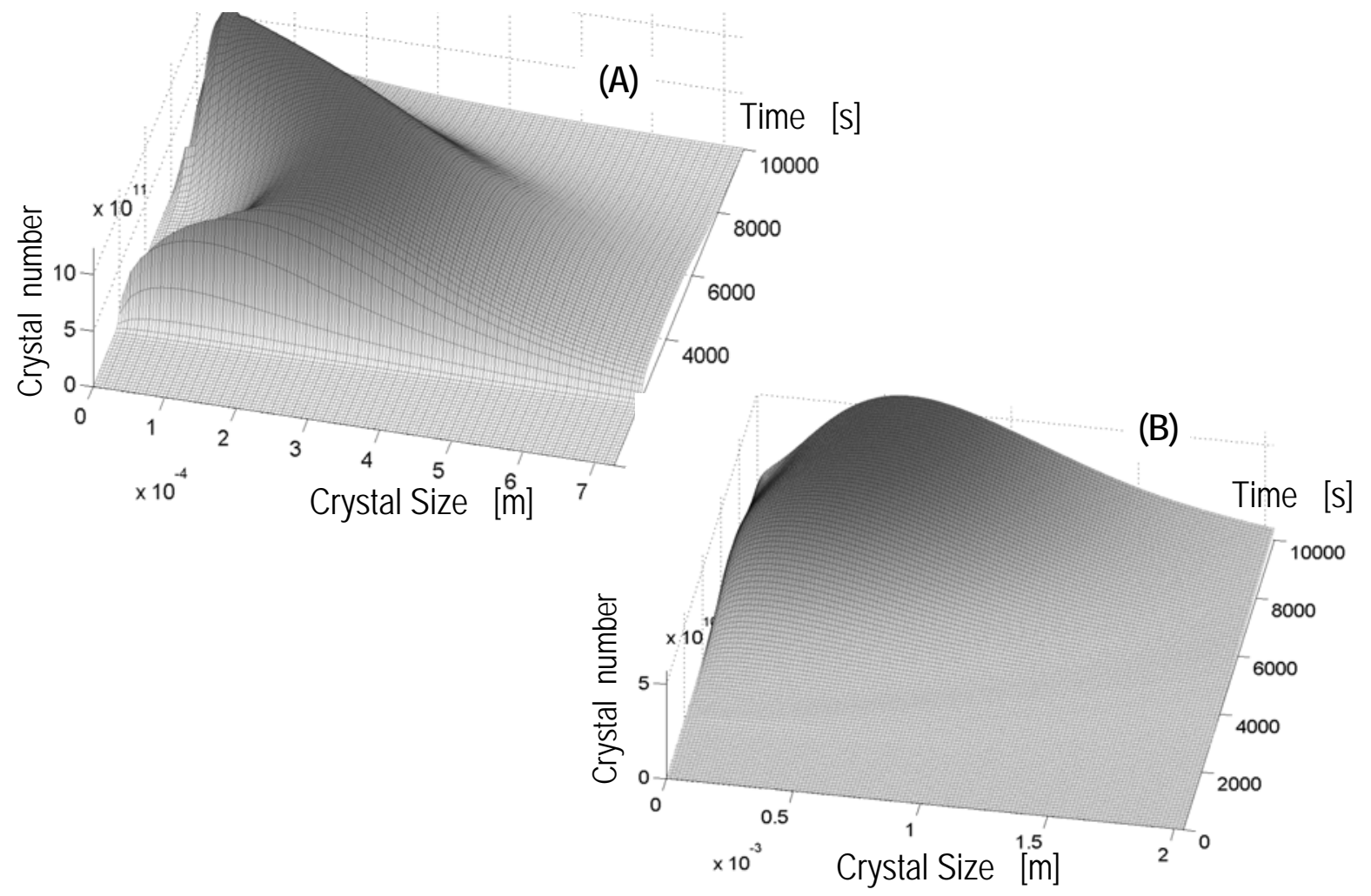

Fig. 5. Time variations of the metastable (A) and Stable (B) Crystal Size Distributions during the crystallization of an API presenting polymorphic transition.
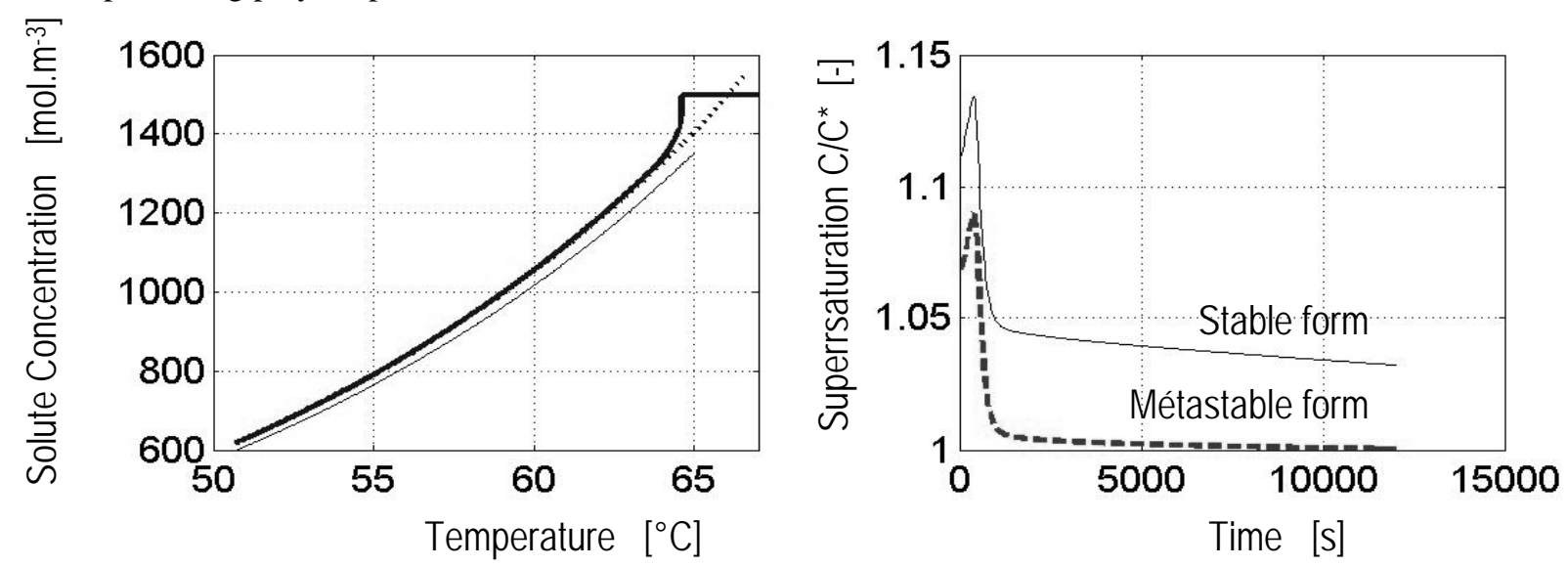

Fig. 6. Simulation of a batch crystallization without solvent-mediated phase transition: the final product is metastable 


\subsection{Simulation of batch crystallization without observable polymorphic transition.}

The kinetic parameters $(\mathrm{A}, \mathrm{B})$ are now tuned so as to increase the initial predominance of the metastable form :

$\mathrm{A}_{1}=3.10^{13} \mathrm{nb} \cdot \mathrm{m}^{-3} \cdot \mathrm{s}^{-1}, \mathrm{~B}_{1}=0.365$

$\mathrm{A}_{2}=2.10^{15} \mathrm{nb} \cdot \mathrm{m}^{-3} \cdot \mathrm{s}^{-1}, \mathrm{~B}_{2}=0.146$.

As one can see in Fig.6, using the same kinetic models as previously, one can simulate situations where the stable product is not obtained at the end of the process. This is of course a realistic situation which clearly demonstrates how metastable forms can be obtained and put the process in jeopardy (for a "historical" similar example see the case of Zantac, (Blagden 1998). When such a product is cropped, no one can say if it is likely, or not, that the stable form will appear further.

\section{CONCLUSIONS \& PERSPECTIVES}

Femlab $^{\circledR}$ appears as a very valuable tool for the modelling of complex chemical engineering processes represented by PDEs. The ease of programming and the efficiency of the software to solve difficult numerical problems, allow one to really save time and, consequently, to focus efforts on improving the physical knowledge of the processes. The phase transition phenomena occurring during the crystallization of APIs are typically problems of significant industrial and theoretical interest. In particular, the dynamic modelling of solvent-mediated polymorphic transitions, which has never been reported before, is a very "hot" topic of research.

After the present study, it seems that actual available nucleation and growth models -provided that they are not too simplistic- allow reproducing the various crystallization behaviours of polymorphs which are observed during the production of complex industrial organic molecules. However, it is important to notice that the simulation results raise many questions and open a wide field for future experimental and theoretical work. For example, it clearly appears from the simulations reported above that the primary nucleation of stable form is unavoidable, even when the Ostwald's Rule of Stage is verified. From a thermo-dynamical point of view, this means that "the fox is set to mind the geese": in presence of stable particles the metastable form should necessarily disappear. In other words, why, if tiny amounts of stable form are inevitably present in the final product, is it so difficult sometimes to forecast the possible occurrence of more stable forms?

Future work of our group will be devoted to deeper analysis of the various situations which can be simulated and to the comparison of computed results with experimental data. In particular, as far as both the concentration profiles and the solid contents can now be measured on-line using ATR FTIR and Raman spectroscopy, respectively, the kinetic parameters introduced above could be estimated. As far as control issues are concerned, the model can be used in the future to design control strategies (e.g. tracking of optimal temperature trajectories) allowing to minimize (or maximize) the generation of a given solid phase. Another major control issue lies in the screening of polymorphs : is it possible to control the process so as to favour the generation of more stable forms and, therefore, to reduce the risk of undesirable generation of unexpected forms during production?

\section{REFERENCES}

Blagden, N., Davey, R. J.,Rowe, R.,Roberts, R. (1998). "Disappearing polymorphs and the role of reaction by-products" Int. J. Pharm. 172(12): 169-177.

Brittain, H. G. (1999). Polymorphism in pharmaceutical solids. New York, Marcel Dekker.

Cambridge (2002). Cambridge Crystallographic Data Center., www.ccdc.cam.ac.uk.

Cardew, P. T., Davey,R.J. (1985). "The kinetics of solvent-mediated phase transformations." Proceedings of the Royal Society of London, Series A: Mathematical, Phys. Eng. Sci. 398(1815): 415-428.

Fevotte, G. (2002). "New perspectives for the online monitoring of pharmaceutical crystallization processes using in situ IR spectroscopy." Int. J. Pharm. 241(2): 263-278.

Garside, J. (1971). "The concept of effectiveness factors in crystal growth." Chem. Engng. Sci. 26: 1425-1431.

Haleblian, J. K. (1975). J. Pharm. Sci. 64: 1269.

Levins, D. M., Glastonbury,J.R. (1972). "Particleliquid hydrodynamics and mass-transfer in a stirred vessel. Part II : Mass transfer." Trans. Instn. Chem. Engrs. 50: 132-146.

Marchal, P. (1989). Génie de la cristallisation : application à l'acide adipique. PhD Thesis, INPL Nancy, France. (in French).

Mersmann, A. (2002). Fundamentals of crystallization. Crystallization Technology Handbook, 2nd Ed. Mersmann. New York., Marcel Dekker

\section{ACKNOWLEDGEMENTS}

The authors are grateful to Dr. Eric Favre for the significant help of comsol-France (Grenoble) during the development of the simulation using Femlab ${ }^{\circledR}$. 